\title{
Racionamiento del agua ante fluctuaciones de disponibilidad. Una discusión teórica para el caso de Chile
}

\author{
Rationing Water and Availability Fluctuations. A Theoretical discussion \\ for the case of Chile
}

Fernando Cabrales . $^{1 *}$, Mauricio Néspolo $C .^{1}$

\begin{abstract}
RESUMEN
El trabajo que se presenta es una discusión teórica sobre las alternativas para racionar un recurso cuando los derechos sobre él exceden la disponibilidad real. Son los conocidos problemas de quiebra. El trabajo es la primera parte de un estudio más amplio sobre el problema, que incluirá levantamiento de información primaria. En zonas como Arica el agua es un recurso natural escaso. Está disponible en cantidades limitadas y frecuentemente debe ser racionada debido a ciclos hidrológicos. Se analiza la ley chilena aplicable al problema, el código de aguas. La escasez relativa del recurso produce efectos importantes sobre las actividades productivas y los períodos de restricción producen costos de eficiencia y efectos distributivos adversos. Las reglas de racionamiento que se estudian son la igualitaria, la proporcional, la de igual pérdida y la regla del Talmud. Las propiedades de las alternativas de racionamiento se explican y analizan axiomáticamente. Se exponen criterios deseables para las reglas de racionamiento. Se concluye que no existe una regla preferible de manera no ambigua y se propone una metodología para evaluar las reglas de asignación en caso de racionamiento.
\end{abstract}

Palabras clave: Regla del Talmud, problemas de quiebra, racionamiento de agua, zonas áridas.

\begin{abstract}
Our work, presented here, is a theoretical discussion on alternatives to ration resource rights when it exceeds the actual availability. They are known as bankruptcy games. The work is the first part of a larger study on the problem, including development of primary information. In zones such as Arica, water is a scarce natural resource. It is available in limited quantities and often should be rationed due to hydrological cycles. Chilean law applicable to the problem is analyzed, the water code. The relative scarcity produces significant effects on productive activities and the restriction periods produce efficiency costs and adverse distributional effects. Rationing rules studied are equalitarian, proportional, the same loss and the Talmud rule. The properties of alternative rationing rules are explained and analyzed axiomatically. Desirable criteria for rationing rules are presented. We conclude that there is no unambiguously better rule and proposes a methodology for evaluating allocation rules in case of rationing.
\end{abstract}

Key words: Talmud rule, arid zones, bankruptcy problems, water rationing.

\section{Introducción}

La disponibilidad de agua para consumo humano, agricultura y procesos industriales es un problema de creciente importancia a escala global. Hay consenso entre los especialistas que este es uno de los mayores desafíos para la humanidad. El problema es aún mayor en zonas áridas, como es el caso de Arica, XV Región de Chile. El caso es especialmente agudo, pues la agricultura es un sector relevante de la actividad económica y el empleo desde el periodo colonial. La manera en que se gestiona el agua en Chile lo define el DFL 1.122 o Código de Aguas y, básicamente, es un sistema de reparto de un recurso de suma cero en el que se conceden derechos de uso sobre la base de estimaciones oficiales de la disponibilidad reconocida. Los derechos concedidos son transferibles libremente y con ello se configura un mercado secundario de derechos. Si hay restricciones de disponibilidad la norma restringe los derechos de manera proporcional a estos. En general el recurso es gestionado por comunidades cuya representación es también proporcional a los derechos que posean las personas. Esta forma de asignación del recurso genera distorsiones en el mercado de derechos, en la actividad productiva y en la distribución de la riqueza

\footnotetext{
1 Escuela de Negocios, Universidad de Tarapacá. Arica Chile.

* Autor para correspondencia: fcabrale@uta.cl
} 
de los involucrados. Por esa razón es necesario evaluar alternativas de racionamiento del agua y un modelo apropiado son los llamados juegos de quiebra. La aplicación de criterios alternativos podría resultar más adecuada para algunos casos, pero puede introducir otros problemas de asignación que no pueden ser resueltos de manera teórica. El trabajo se organiza de la siguiente manera; en la primera sección se analiza la norma chilena para el uso de agua. En la segunda sección se analizan los efectos económicos de la norma. En la tercera se analizan axiomáticamente las normas alternativas de reparto y en la cuarta se propone una metodología para contribuir a valorar y elegir una norma y se concluye.

\section{La Gestión del Agua en Chile}

Las aguas terrestres son bienes nacionales de uso público y el DFL 1.122 de 1981 otorga a los particulares el derecho de aprovechamiento de ellas, en conformidad a las disposiciones del denominado Código de Aguas. Las normas de uso son análogas para el caso de aguas corrientes o estancadas, subterráneas o superficiales. Los derechos de agua son libres y gratuitos cuando se solicitan a la autoridad administrativa. Se expresan en unidades de flujo y asociadas a una fuente o cauce natural de la que se extrae. Sin embargo una vez que la autoridad ha concedido un derecho de aprovechamiento, este es transable. Legalmente se trata como un bien mueble, independiente de la propiedad territorial, y se puede transferir o vender libremente.

La autoridad administrativa, la Dirección General de Aguas (DGA), debe cumplir y hacer cumplir ciertos requisitos para la concesión de derechos de extracción o aprovechamiento de aguas. Debe asegurar la publicidad de las solicitudes (para dar la oportunidad de oposiciones y evitar duplicidades de derechos) y puede otorgar derechos solo cuando exista certeza de la disponibilidad de los flujos solicitados. Dicha disponibilidad se traduce en la fijación de un máximo posible de extracción desde una fuente natural (cauce, acuífero o fuente). Dicho máximo se debe constituir preservando mínimos ecológicos que impidan la degradación ambiental de la zona de extracción (artículo 129 bis, DFL 1.122).

De esa manera se constituyen mercados zonales de derechos de agua. Dichos mercados están asociados a la fuente de extracción, es decir, cuenca hidrográfica, curso natural, acuífero subterráneo u otra.
La administración de la infraestructura de distribución y conservación de las aguas suele estar en manos de comunidades de agua. Salvo en los casos en que el recurso es muy abundante, la construcción de canales, embalses, canales de distribución, inspección y control está en manos de estas asociaciones. Estas comunidades tienen un sistema de representación proporcional a los derechos de extracción (acciones) que cada individuo posea. La comunidad de aguas se gestiona, entonces, de manera similar a una sociedad anónima.

En caso de sequía o escasez, los derechos de agua se racionan de manera alícuota entre los poseedores de derechos de extracción (artículo 17, DFL 1.122). Este criterio se extiende al caso de solicitudes de usuarios que se vean perjudicados por extracciones mayores que la disponibilidad (artículo 62, DFL 1.122). La DGA puede decretar zonas de restricción para el uso de agua (artículo 65, DFL 1.122) y en esos casos deben constituirse comunidades de aguas, las que deben aplicar a prorrata de los derechos la restricción aplicada.

En casos extremos de sequía (artículo 313, DFL 1.122), calificados por la DGA, el Presidente de la República puede declarar una zona de sequía hasta por seis meses no prorrogables. En esos casos, la DGA puede autorizar extracciones de agua en los puntos que estime convenientes y sin mínimos (aun bajo el mínimo ecológico). Los comuneros afectados por una declaración de ese tipo deben ser indemnizados por el Estado.

En casos de expropiación de derechos de extracción para satisfacer necesidades de consumo doméstico a la población, la autoridad debe dejar al expropiado los derechos necesarios para su propio consumo doméstico (artículo 27, DFL 1.122).

De esa manera, el legislador ha adoptado un criterio proporcional de reparto ante escasez. Esta proporcionalidad está acotada en el límite del consumo doméstico de un poseedor de derechos de agua. Además aparece una situación de discrecionalidad absoluta (aunque indemnizable) en caso de sequía severa.

\section{Consecuencias Económicas del Sistema de Reparto en caso de Sequía}

Los derechos transables de extracción de agua y la consecuente conformación de un mercado competitivo de derechos aseguran que, en ausencia de distorsiones, la asignación de recursos sea 
óptima. Los usuarios que dispongan de derechos menos productivos terminarán por vender dichos derechos a quienes tengan usos más productivos. La homogeneidad del bien asegura que el mercado no tiene asimetrías de información en lo que se refiere al producto. El precio de los derechos de agua, fluctuando libremente, puede despejar el mercado, de modo que no exista escasez. Hay, sin embargo, algunas distorsiones que están vinculadas a la escasa capacidad de fiscalización de la DGA. Esta dificultad puede permitir la extracción ilegal de aguas (especialmente subterráneas) sin consecuencias importantes para el infractor. También se pueden interpretar algunas situaciones como de "captura" del regulador, pues la importancia política del agua puede relajar las normas de sustentabilidad del recurso.

Las fluctuaciones cíclicas de la disponibilidad de agua producirán efectos de riqueza que pueden resultar injustos desde el punto de vista distributivo. También pueden producir costos de eficiencia desde el punto de vista productivo.

En un modelo simple de un recurso disponible en una cantidad fija, los cambios en la oferta del bien producen rentas especulativas. Alternativamente permitirá que aquellos con mayor capacidad financiera puedan especular con los derechos. Desde el punto de vista de las actividades productivas, un período de sequía (una fluctuación cíclica) puede producir efectos permanentes si es que aquellas empresas menos rentables venden sus derechos de uso del recurso.

Ciertamente lo descrito puede evitarse con un mercado de seguros eficiente. Sin embargo los seguros de este tipo no existen en Chile.

Además, si se considera que las comunidades de aguas operan de manera análoga a una sociedad anónima, es decir, toman decisiones basadas en las preferencias de aquellos que poseen más derechos, entonces aparece la posibilidad de capturas hostiles, perjuicios de minoritarios y otras deficiencias societarias ${ }^{1}$. En general estas están suficientemente reguladas en el caso de las sociedades por acciones, pero la regulación de los derechos de agua como instrumento de decisión al interior de las comunidades de aguas es débil y no sanciona este tipo de conductas. La posibilidad de control monopólico del agua en amplias zonas geográficas es un peligro latente con las consecuencias fáciles de prever. Las fluctuaciones cíclicas de la asignación del recurso abren la posibilidad a este control monopólico si es que un grupo de usuarios es relativamente pobre y utiliza el agua en actividades menos rentables ${ }^{2}$. En esos casos el costo alternativo del recurso puede superar la rentabilidad de la actividad que desarrollan.

Es posible preguntarse si hay reglas de reparto del recurso que sean más equitativas desde el punto de vista distributivo y más eficientes desde el punto de vista económico que la que adopta la ley chilena ante fluctuaciones cíclicas en la disponibilidad del recurso. También es necesario conocer las características y las consecuencias de esas reglas alternativas de reparto.

\section{Reglas de Reparto}

El problema de la asignación de recursos escasos entre usos alternativos es de hecho parte de la definición de ciencia económica. La economía de mercado tiene un método de racionamiento: el sistema de precios. Son los precios que enfrentan los agentes económicos, las restricciones (presupuestarias, de tiempo u otras) y las preferencias individuales las que priorizan los bienes o servicios que los agentes deciden consumir y, de una manera dual, definen el costo económico como el sacrificio (costo de oportunidad) que se hace al dejar de consumir otros bienes.

Una clase especial de problema que se puede presentar es el de incumplimiento de compromisos (tácitos o explícitos), son los llamados problemas de quiebra ${ }^{3}$. Este tipo de problemas escapa a las reglas del mercado, pues se trata de situaciones en las que existen compromisos que no se pueden cumplir. De hecho el nombre deriva del problema que se produce cuando una empresa quiebra y debe repartir el valor de los activos y derechos que posee, entre deudas que superan el valor de la empresa. Como es obvio el "intercambio libre" del mercado no es aplicable al caso, pues un agente no cumplirá con sus compromisos. Sin embargo, en un nivel más general, hay muchas otras razones que impiden el intercambio libre del mercado y hacen necesaria la aplicación de reglas de reparto exógenas. Por ejemplo la asignación del presupuesto público es una decisión, casi por definición, política. El reparto de una herencia, el uso de recursos comunes, y muchos otros, son problemas en los que el mercado tiene poco que decir.

En esos casos las reglas de reparto deben proporcionar una distribución "apropiada" de la 
cantidad a repartir considerando los derechos que esgrimen los agentes.

La solución práctica a problemas de bancarrota tiene muchas variantes y eso obliga a tratar cada caso de manera específica al problema. Por ejemplo, en el caso de la Ley de Quiebras chilena, existen prelaciones para la recuperación de compromisos. Así, las deudas tributarias, las que involucran garantías hipotecarias y las previsionales tienen prioridad sobre las remuneraciones, las facturas impagas y otras acreencias. En el caso de las herencias existe solo un margen que puede testarse libremente, en el caso de la ejecución de deudas impagas hay bienes muebles que son inembargables (Hougaard et al., (2013) teorizan el caso en que la quiebra debe considerar un mínimo a asignar a cada agente). En el caso de la distribución del presupuesto público, hay múltiples criterios que se aplican al reparto de recursos escasos. Además, los derechos (deudas, necesidades, obligaciones, u otras) pueden ser de diversa naturaleza (deudas, demandas, rentas o necesidades, en los ejemplos anteriores). Consecuentemente la forma más adecuada de resolver un problema de bancarrota dependerá en general del tipo de problema de que se trate y no solo de la información resumida en la cantidad del bien a repartir y el vector de reclamaciones. Este tipo de ejercicios para casos específicos los han realizado Ansink y Weikard (2009 y 2012), Madani y Dinar (2013), Mianabadi et al. (2013).

\subsection{Las reglas básicas de reparto en problemas de quiebra}

En esta sección se caracterizarán reglas genéricas de reparto bajo supuestos simplificadores que permitan entender adecuadamente las alternativas que existen para este tipo de problemas. Para ello utilizaremos el llamado enfoque axiomático de las reglas de reparto 4 . Las reglas básicas son:

(i) La regla proporcional, que divide los recursos a repartir de manera proporcional a los derechos de los agentes. Es la regla general del código de aguas.

(ii) La regla igualitaria, que reparte por igual los recursos disponibles.

(iii) La regla de igual pérdida, que reparte igualitariamente la pérdida total.

(iv) La regla del Talmud, que es una combinación de la regla igualitaria y de la de igual pérdida y que explicamos con más detalle.
A continuación presentamos un modelo general del problema con algunas expresiones formales que extrajimos de Moreno-Ternero y Villar (2004) y presentamos las cuatro soluciones al problema de bancarrota. A continuación extractamos algunas propiedades que estimamos importantes. Específicamente explicamos cinco teoremas que caracterizan las reglas de reparto que se obtienen también de Moreno-Ternero y Villar (2004). La tercera explica con un ejemplo numérico simplificado las soluciones que se pueden obtener. En esta sección incluimos comentarios sobre la manera cómo se debieran evaluar las alternativas de reparto.

\subsection{El problema general de reparto}

Supongamos $\mathrm{n}$ agentes (personas, familias, empresas o incluso sectores industriales como agricultores, mineros, industriales). Cada uno de estos tiene un derecho $c_{i}$ sobre una parte de un bien (agua, tierras, dinero, u otro recurso divisible). Digamos que E es la cantidad disponible del bien, en circunstancias que la suma de los derechos es mayor que E. Supongamos que los derechos no son calificables, es decir, los títulos que los respaldan tienen el mismo valor y que los agentes son todos iguales entre si de manera que el reparto no puede discriminar entre ellos. La regla de reparto debe ser imparcial y por ello solo se consideran la cuantía de los derechos y la cantidad total del bien a repartir. Se supone que se aplicarán criterios éticos y procesales que permitan repartir E solo basándose en los derechos.

El problema de quiebra es distributivo y consiste en el reparto de $\mathrm{E}$, que es divisible, entre un conjunto $\mathrm{N}=\{1,2, \ldots, \mathrm{n}\}$ de agentes, en circunstancias que la disponibilidad del recurso no alcanza para satisfacer todos los derechos expuestos: $\mathrm{c} 1, \mathrm{c} 2, \ldots, \mathrm{cn}$. Como es evidente $\mathrm{E}>0$, y representaremos los derechos de los agentes con un vector $c \in \mathbb{R}_{+}^{n}$. Así, un problema de quiebra puede resumirse mediante un par $(\mathrm{E}, \mathrm{c})$ en $\mathbb{R}_{+}^{n}$, en que $\Sigma \mathrm{c}_{\mathrm{i}}<\mathrm{E}$.

Geométricamente el problema puede mostrarse como en el Figura 1, en el caso de dos agentes. En los ejes tenemos las reclamaciones de los agentes y representamos la disponibilidad del bien mediante la línea E. La suma de los derechos (punto C, la suma de las demandas) está fuera de la restricción presupuestaria (Figura 1).

Denominamos Bn la familia de problemas de bancarrota con $n$ agentes. C será la reclamación 


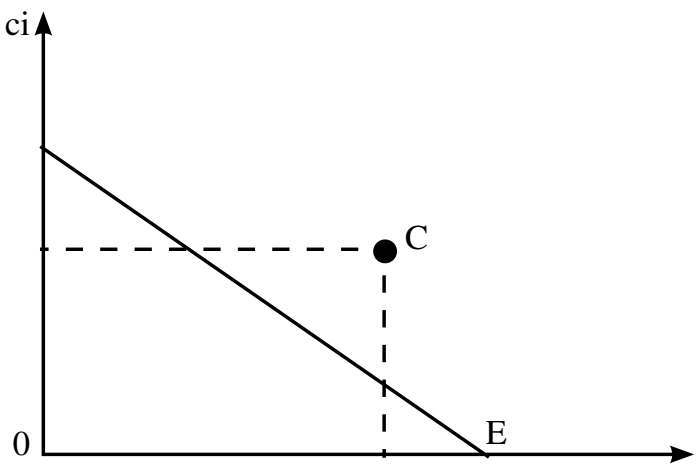

Figura 1. Problema de quiebra para dos agentes.

agregada y la diferencia $\mathrm{C}$ - E $>0$ representa el déficit o la pérdida colectiva del problema de quiebra (E,c).

Una solución a un problema de quiebra con $n$ agentes es una función $\mathrm{F}$ que a cada problema (E,c) de $\mathrm{Bn}$ asocia un único punto $\mathrm{F}(\mathrm{E}, \mathrm{c}) \mathbb{R}_{+}^{n}$ tal que:

(i) $\mathrm{c} \geq \mathrm{F}(\mathrm{E}, \mathrm{c}) \geq 0$.

(ii) $\Sigma \mathrm{c}_{\mathrm{i}} \mathrm{Fi}(\mathrm{E}, \mathrm{c})=\mathrm{E}$.

El punto $F(E, c)$ es la forma apropiada de distribuir E de acuerdo con la regla de reparto. La condición (i) dice que nadie obtiene más de lo que reclama y percibe una parte no negativa del bien. La condición (ii) indica que se reparte todo el bien disponible.

Las tres primeras soluciones, proporcional, igualitaria y de igual pérdida, aplican un principio igualitario sobre variables diferentes entre sí. La proporcional, iguala la proporción entre la cantidad obtenida y cantidades reclamadas respecto del total obtenido y reclamado, respectivamente. La segunda iguala la cantidad obtenida por cada agente. La tercera equipara las pérdidas respecto de los derechos esgrimidos. La regla del Talmud aplica una regla compuesta que hace que ningún agente obtenga más de la mitad de lo reclamado si la cantidad del bien es inferior a la mitad de la cantidad total reclamada. Pero si el bien o recurso supera a la mitad del total de derechos, ningún agente pierde más de la mitad de lo demandado. Veamos más detenidamente cada una de estas reglas.

\subsection{Las soluciones igualitarias}

La solución proporcional es la forma más conocida y utilizada de resolver problemas de quiebra. De hecho es la que aplica el código de aguas chileno.
Formalmente el reparto proporcional $(\mathrm{P})$ es aquella regla que aplica a cada problema $(\mathrm{E}, \mathrm{c}) \mathrm{de}$ $\mathrm{Bn}$ una asignación $\mathrm{P}(\mathrm{E}, \mathrm{c})=\lambda \mathrm{c}$, donde $\lambda=\mathrm{E} / \mathrm{c}$.

El reparto igualitario (I) propone resolver los problemas de quiebra igualando las cantidades percibidas por todos los agentes. Se agrega la restricción de que nadie perciba más de lo que reclama. Formalmente la definimos como aquella solución en la que para cada problema (E,c) de Bn y para cada agente $\mathrm{i}=1,2, \ldots, \mathrm{n}$ asigna recursos a cada individuo: $\operatorname{Ir}(\mathrm{E}, \mathrm{c})=\min \{\lambda \mathrm{cj}\}, \lambda$ es solución de la ecuación $\Sigma \mathrm{c}_{\mathrm{i}} \min \{\lambda, \mathrm{cj}\}=\mathrm{E}$.

La regla de reparto de igual pérdida (IP) distribuye igualitariamente la diferencia entre la cantidad total demandada y la cantidad del bien disponible. Es decir, cada agente percibe una cantidad $x_{j}=c_{j}-C-E / n$. Aplicamos una restricción no negatividad $\left(x_{i} \geq 0\right)$, porque puede ocurrir que la aplicación de la regla lleve a soluciones negativas para algunos demandantes. Geométricamente esta solución a los problemas de bancarrota es intuitiva, porque equivale a elegir el punto de la restricción presupuestaria más cercano al vector de demandas. Formalmente la solución IP es aquella que para un problema $(\mathrm{E}, \mathrm{c})$ de $\mathrm{Bn}$, asigna para cada $\mathrm{i}=1,2, \ldots, \mathrm{n}$ :

$\operatorname{IP}_{\mathrm{i}}(\mathrm{E}, \mathrm{c})=\max \left\{0, \mathrm{c}_{\mathrm{i}}^{*} \mu\right\}$ con $\mu>0$ y es solución de la ecuación $\Sigma \mathrm{c}_{\mathrm{i}} \max \left\{0, \mathrm{c}_{\mathrm{i}}-\mu\right\}=\mathrm{E}$.

En el Figura 2 se ilustran las asignaciones de las tres soluciones. En el Figura 2(a) aparecen las soluciones que las reglas determinan ante un caso de quiebra con los parámetros indicados. En el Figura 2(b) se muestra la asignación que se obtiene en cada regla, dado un vector de reclamaciones, pero variando el presupuesto de $0 \mathrm{a} \mathrm{C}$.

\subsection{La regla del Talmud ${ }^{5}$}

Aumann y Maschler (1985) analizan una regla de reparto del Talmud referida al reparto de una herencia cuyo valor no alcanza la suma propuesta en el testamento. Los rabinos proponen valores de reparto cuya expresión axiomática no está explícita. Descubren que los rabinos aplican un criterio distributivo compuesto que depende de la relación entre la cantidad del bien disponible (E) y la mitad de las reclamaciones sumadas $(\mathrm{C} / 2)$. La regla del Talmud aplica una idea de protección a los agentes en la que la pérdida de cada agente es análoga a la pérdida agregada. En esta regla de reparto ningún agente obtiene más de la mitad de su deuda cuando los recursos disponibles son 

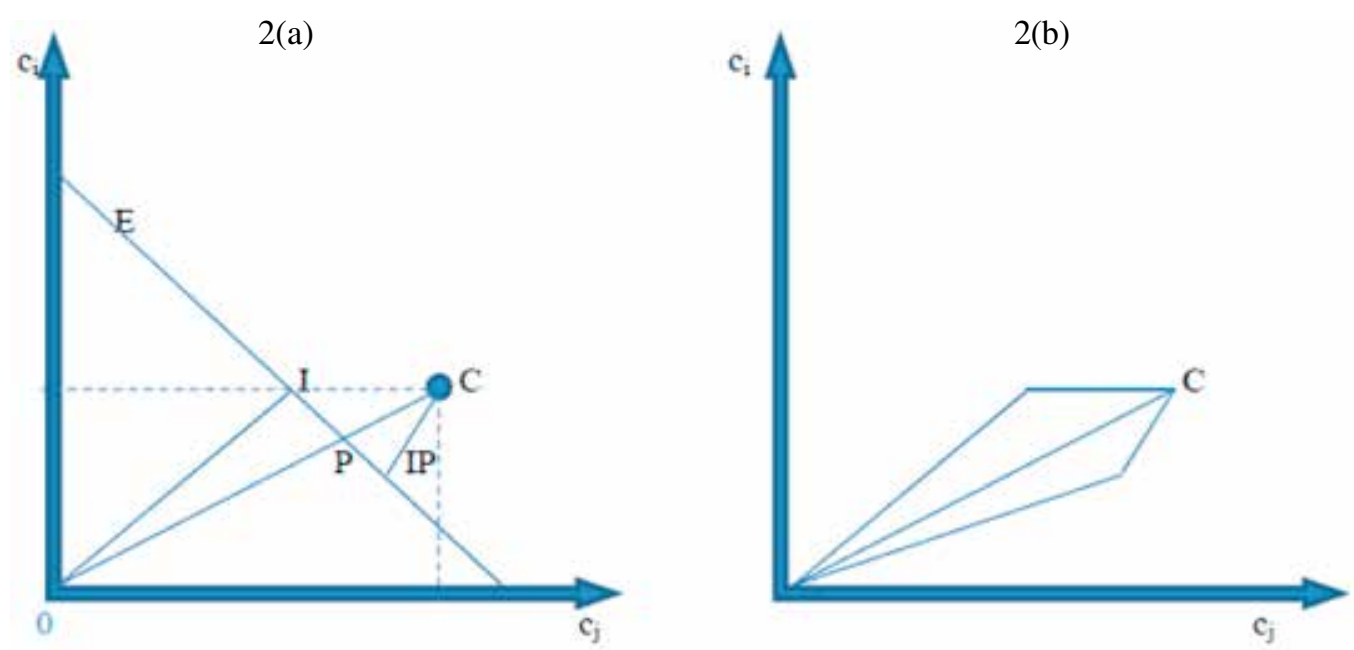

Figura 2. Soluciones de las diferentes reglas en caso particular con C, E y en el caso que se haga variar la restricción desde 0 a C.

menores que la mitad de la deuda agregada. Los agentes tampoco perderán más de la mitad de su demanda si los recursos disponibles superan la mitad de la demanda agregada. Formalmente la regla del Talmud (T) se define como aquella que asigna a cada problema (E,c) de Bn, y para cada $\mathrm{i}=1,2, \ldots$, $\mathrm{m}$, las siguientes cantidades del recurso divisible:

$$
T_{i}(E, c)=\begin{aligned}
& \min \left\{\frac{1}{2} c_{i}, \lambda\right\} \text { si } E \leq \frac{C}{2} \\
& \max \left\{\frac{1}{2} c_{i}, c_{i}-\right\} \text { si } E \geq \frac{C}{2}
\end{aligned}
$$

donde $\lambda, \mu>0$ son solución de las siguientes ecuaciones:

$\Sigma c_{i} \min \left\{1 / 2 c_{i}, \lambda\right\}=E$ y $\Sigma c_{i} \max \left\{1 / 2 c_{i}, c_{i}-\mu\right\}$.

Gráficamente, considerando un vector de demandas dado, y una cantidad variable (desde 0 a C) del recurso a repartir es como sigue (Figura 3):

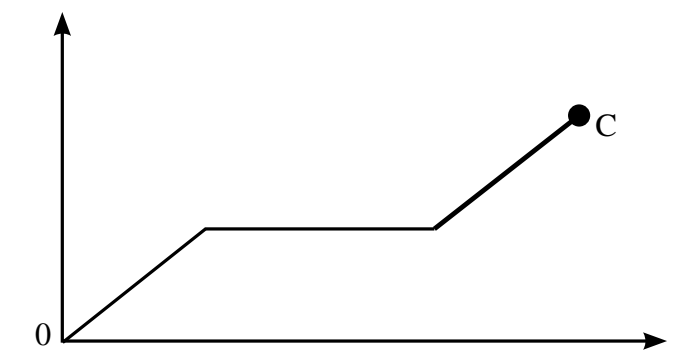

Figura 3. Solución del Talmud con cantidad variable de recursos a repartir.
Aumann y Maschler (1985) prueban que esta es la única regla de reparto que extiende de manera consistente la solución propuesta para el problema de reparto, cuando la aplicamos a más de dos agentes. Es decir, al caso general de "n" demandantes. La "consistencia" quiere decir que si aplicamos la regla de reparto a un problema con $n \geq 2$ agentes, el resultado será el mismo que si la aplicamos a subconjuntos de dos agentes, tomando como demandas las originales y como cantidad a repartir la que se les asignó en el problema inicial.

\subsection{Algunas propiedades de las reglas de reparto}

La calidad de las reglas de reparto en los problemas de quiebra puede evaluarse si cumplen ciertos requisitos. Idealmente interesaría que las reglas fueran consistentes en el sentido del párrafo anterior. Pero también es importante que sean objetivas y claras para todo el mundo. En realidad se pueden exigir muchos requisitos a las reglas de reparto, algunas de las cuales reseñamos más adelante. Pero hay algunas propiedades incompatibles entre sí y en ese sentido hay que optar entre las cualidades de una regla respecto de las de otra. A continuación presentamos algunas que estimamos importantes. En nuestro trabajo de investigación argumentamos en favor de otro procedimiento (que se explica más adelante) y por ello se presentan sin mayores demostraciones. 
i. Tratamiento igualitario: El tratamiento igualitario es un requisito ético. Decimos que si los demandantes son iguales, deben ser tratados de la misma manera. En el caso sencillo significa que demandas iguales deben acceder al mismo resultado.

ii. Independencia de la escala: Las soluciones de reparto debieran ser independientes de las unidades de medida. En el caso del agua, si expresamos un problema en distribuir litros por segundo, la solución obtenida debiera ser igual a la que se obtenga cuando se trabaja en metros cúbicos por hora.

Las siguientes propiedades son importantes para el caso de un recurso como el agua. Supongamos que ha habido algún error de proyección de la cantidad de agua disponible. Dicho error se descubre después de fijar una distribución cualquiera y el error puede ser por defecto o por exceso. Es decir, se distribuyó menos agua que la que finalmente hubo o, alternativamente, se distribuyó más que la que existía. En una situación como esta, hay al menos dos alternativas de acción: (a) Resolver nuevamente el problema con los datos verdaderos o (b) Realizar un ajuste a la distribución inicial. Ambos procedimientos debieran llegar al mismo resultado. A estas propiedades las denominamos:

iii. De composición hacia arriba.

iv. De composición hacia abajo.

v. Consistencia: La consistencia se explicó antes, se refiere al caso de un conjunto de reclamantes variable. La propiedad exige que la regla de reparto entregue los mismos resultados si se aplica a todos los participantes que si se aplica sobre subconjuntos de los reclamantes. Esta propiedad elimina los incentivos a discutir indefinidamente una distribución con parámetros iguales, pues la solución no se verá alterada.

Las soluciones proporcional, igualitaria e igual pérdida cumplen con estas cinco propiedades. Moulin (2000) demuestra que, de hecho, solo las soluciones I, P, IP satisfacen las cinco propiedades. La demostración es importante porque significa que otras reglas de asignación violarán algunos de los criterios anteriores.

Sin embargo hay criterios adicionales que producen diferencias entre las reglas.

vi. Autodualidad: La solución de un problema de quiebra puede entenderse como el reparto de una cantidad $\mathrm{E}$ en función del vector de demandas c, y también como el reparto de una pérdida (C - E) en función del mismo vector. Es decir, a cada solución $F$ podemos asociarle una asignación dual $\mathrm{F}^{*}$ del siguiente modo:

$\mathrm{F}^{*}(\mathrm{E}, \mathrm{c})=\mathrm{c}-\mathrm{F}(\mathrm{C}-(\mathrm{E}, \mathrm{c}))$ En que $(\mathrm{E}, \mathrm{c})$ denota la solución primaria.

Si una regla de reparto satisface la propiedad de autodualidad significa que la solución al reparto del recurso, y su dual, es decir, la regla de reparto de la pérdida, coinciden.

La regla de proporcionalidad y la regla del Talmud son autoduales. Young (1987 y 1988) demuestra que la única solución que verifica las propiedades de composición hacia arriba y autodualidad es la proporcional.

Las propiedades de exención, exclusión y aseguramiento aplican criterios de compensación asociados a las demandas individuales relativas al bien disponible. Son usuales en muchos problemas prácticos de quiebra y son duales.

vii. Exención: Exige que si los recursos a repartir son relativamente grandes, solo los agentes con reclamaciones mayores deben ser racionados. Puede asimilarse al principio general de "progresividad" en el que los agentes con reclamaciones menores son prioritarios en el reparto. Específicamente si un agente reclama menos de la cantidad disponible per cápita, entonces debe recibir todo lo que demanda.

Un ejemplo de la aplicación del principio son las normas de seguros de depósitos que existen en muchos países que protegen al pequeño ahorrista. Hay varias razones para esta protección entre las que mencionamos: (a) Es posible que los ahorros menores representen una proporción mayor de la riqueza de los ahorrantes. (b) Están más indefensos frente a litigios onerosos. (c) Tiene un efecto agregado sobre el comportamiento de los ahorrantes en general. (d) Los ahorros pueden estar destinados a consumos más importantes socialmente (vivienda, educación de hijos, consumo en la etapa de retiro).

viii. Exclusión: Es un juicio de valor opuesto al de exención, es decir, las reclamaciones muy pequeñas deben ser ignoradas. De hecho podemos aplicarla diciendo que si un agente tiene una demanda inferior al déficit per cápita, entonces debe perder toda su reclamación. El juicio de valor implícito en este principio puede surgir de considerar muy importante (vital) el recurso a repartir. Pensemos en el caso de una catástrofe en la que hay personas que requieren 
mucha atención médica, alimentación, refugio $\mathrm{u}$ otros recursos parecidos y otros agentes cuyo déficit es menor (sufrieron daños personales o patrimoniales menores).

La regla igualitaria (I) verifica la propiedad de exención y no exclusión. Al revés, la regla de igual pérdida (IP) verifica exclusión pero no exención. La regla proporcional no verifica ninguna de las dos propiedades. Además Herrero-Maschler y Villar (2001) prueban que solo la regla igualitaria satisface las propiedades de composición hacia abajo, exención y consistencia.

También los autores prueban que solo la regla de igual pérdida (IP) cumple las propiedades de composición hacia arriba, exclusión y consistencia. ix. Aseguramiento: Las propiedades anteriores descansan en un juicio de valor opuesto y extremo respecto de agentes con reclamaciones pequeñas. El aseguramiento es un criterio moderado que garantiza pagos mínimos a los agentes con demandas menores. Diremos que una regla de reparto cumple con el criterio de aseguramiento si, siendo su demanda menor que el total de recursos disponibles, recibirá al menos su $c_{i} / n$ y si su demanda es mayor que el total de recursos disponibles recibirá al menos E/n.

En esta solución el pago garantizado para cada agente depende de su propia reclamación, el número de agentes implicados en el problema y la cantidad a repartir. Es, de hecho, independiente de las demandas de los otros agentes. Así, si una regla de reparto cumple con esta propiedad, el agente sabrá la compensación mínima, con independencia de las de los demás agentes. La solución de igual pérdida (IP) y la regla del Talmud (T) satisfacen esta propiedad. Además Moreno-Ternero y Villar (2004) demuestran que solo la regla del Talmud satisface consistencia, aseguramiento y autodualidad.

\section{El Problema de Evaluación de las Reglas de Quiebra}

Es fácil advertir que el Código de Aguas de nuestro país opta por una regla de reparto que podría ser mejorada sustancialmente en especial si incorporamos cualquiera de los criterios desde el (vi) en adelante. También parece evidente que el caso del agua en el desierto más árido del mundo (Arica) debiera incorporar algunos criterios éticos de reparto en caso de fluctuaciones en la disponibilidad del recurso. Finalmente, si observamos que la regla del Talmud satisface las propiedades de consistencia, aseguramiento y autodualidad, resulta atractiva de incorporar a la legislación chilena.

Sin embargo la posibilidad de incorporar la regla del Talmud al código de Aguas encuentra dos problemas relevantes y relacionados.

1. La regla del Talmud no satisface los requisitos de composición hacia arriba y hacia abajo, es decir, el reparto del recurso puede variar si hay cambios inesperados (por exceso o por defecto) del recurso. Ello introduce un problema de gestión ante cambios en la disponibilidad del recurso, pues aparecen incentivos para revisar continuamente el reparto obtenido con un determinado set de información.

2. En una sociedad democrática, los juicios de valor se resuelven mediante un tipo especial de juego, la votación. Así, aunque las propiedades de la regla del Talmud sean atractivas, no son definitivas. Por ejemplo, si nos parece atractiva la propiedad de aseguramiento (recordamos una regla intermedia entre exclusión y exención), habrán personas a las que les parezca que el agua es demasiado importante y consideren que la regla de exclusión debiera imponerse, al menos en casos de sequías muy severas.

Ambas dificultades impiden tomar decisiones al respecto e introducen incentivos a la revisión de los resultados de asignación de la regla que se aplique. A continuación mostramos un ejercicio numérico hipotético de una situación de sequía, en el que dos agricultores tienen derechos de extracción por $\mathrm{c}_{\mathrm{a}}=60 \mathrm{~L} / \mathrm{s} \mathrm{y} \mathrm{c}_{\mathrm{b}}=40 \mathrm{~L} / \mathrm{s}$. Ahora supongamos tres situaciones de restricción de agua. La disponibilidad es de $90 \mathrm{~L} / \mathrm{s}$, la disponibilidad es de $50 \mathrm{~L} / \mathrm{s}$ y la disponibilidad es de $20 \mathrm{~L} / \mathrm{s}$. La Tabla 1 muestra las asignaciones si aplicamos la regla proporcional (P, del actual Código de Aguas), la igualitaria (I), la de igual pérdida (IP) y la regla del Talmud (T).

Tabla 1. Resultados de asignación entre agentes (a,b) con distintas reglas.

\begin{tabular}{lccc}
\hline Método & $\mathrm{E}=90 \mathrm{~L} / \mathrm{s}$ & $\mathrm{E}=50 \mathrm{~L} / \mathrm{s}$ & $\mathrm{E}=20 \mathrm{~L} / \mathrm{s}$ \\
\hline $\mathrm{P}$ & $(54 ; 36)$ & $(30 ; 20)$ & $(12 ; 8)$ \\
$\mathrm{I}$ & $(45 ; 45)$ & $(25 ; 25)$ & $(10 ; 10)$ \\
$\mathrm{IP}$ & $(55 ; 35)$ & $(35 ; 15)$ & $(20 ; 0)$ \\
$\mathrm{T}$ & $(55 ; 35)$ & $(30 ; 20)$ & $(10 ; 10)$ \\
\hline
\end{tabular}

Como se observa, la regla del Talmud se equipara a la de Igual Pérdida si la restricción es moderada. 
Equivale a la proporcional si la restricción es mediana y a la igualitaria si la restricción es severa.

¿Cómo se puede saber cuál es la "mejor" regla de reparto? Aun cuando el problema es muy sencillo, no se pude resolver sin un procedimiento específico compatible con una sociedad democrática. Incluso si se decidiera llevar a una votación, la "imposibilidad de Arrow" enseña que no es posible traducir las preferencias individuales a una preferencia colectiva sin violar algunos supuestos de consistencia democrática.

Estimamos que este problema puede aminorarse con técnicas de valoración contingente (MVC). La MVC se utiliza para estimar los cambios en el bienestar colectivo ante cambios cuantitativos y cualitativos que se producen de manera conjunta. De hecho se utiliza frecuentemente para estimar el valor de los cambios ambientales que se producen con la realización de proyectos de inversión.
El método es indirecto, pues intenta medir, mediante entrevistas, la valoración individual de un cambio propuesto. La valoración se conoce como la Disposición a Pagar (DAP), que se expresa en dinero $\mathrm{y}$, por eso, permite establecer comparaciones entre alternativas. Si recordamos que los derechos de agua son transferibles, entonces es posible interrogar a propietarios de derechos sobre su disposición a obtener los derechos que les faltarán en caso de restricción. Observando la Tabla de ejemplo, es evidente que la valoración del método de racionamiento será distinta ante distintas intensidades de restricción. Sin embargo las preguntas se pueden hacer en diversos escenarios de restricción, de manera que se pueden obtener valoraciones completas de cada regla.

La investigación futura sigue ese camino. La idea es desarrollar un cuestionario que será sometido a la consideración de agentes diversos, propietarios de derechos de agua, en la región de Arica y Parinacota.

\section{Literatura Citada}

Ansink, E.; Weikard, H.P.

2012. Sequential sharing rules for river sharing problems. Soc Choice Welf, 38: 187-210.

Ansink, E.; Weikard, H.P.

2009. Contested water rights. European Journal of Political Economy, 25: 247-260.

Aumann, R.; Maschler, M.

1985, Game Theoretic Analysis of a Bankruptcy Problem from the Talmud. Journal of Economic Theory, 36: 195-213.

Azqueta, D.

1994. Valoración Económica de la Calidad Ambiental. Mc Graw- Hill, Madrid, Epaña.

Biblioteca del Congreso Nacional. DFL 1.122 http://www. leychile.cl/Navegar?idNorma $=5605 \&$ buscar $=$ codigo + de + aguas, consultado 20 de enero de 2014.

Herrero, C.; Maschler, M.; Villar, A.

2001, The Three Musketeers: Four Classical Solutions to Bankruptcy Problems. Mathematical Social Sciences, 42: 307-328.

Hougaard, J.L.; Moreno-Ternero, J.D.; Østerdal, L.P.

2013. Rationing in the presence of baselines. Soc Choice Welf, 40: 1047-1066.
Madani, K.; Dinar, A.

2013. Exogenous regulatory institutions for sustainable common pool resource management: Application to groundwater. Water Resources and Economics, 2-3: 57-76.

Mianabadi, H.; Mostert, E.; Zarghami, M.; Van de Giesen, N. 2013. Transboundary water resources allocation using bankruptcy theory; Case study of Euphrates and Tigris Rivers, en Proceedings of the TWAM2013 International Conference \& Workshops, pp. 1-5.

Moreno-Ternero, J.D.; Villar, A.

2004. The Talmud Rule and the Securement of Agents' Awards, Mathematical Social Sciences, 47: 245-257.

Moulin, $\mathrm{H}$

2000. Priority Rules and Other Asymmetric Rationing Methods. Econometrica, 68: 643-684.

Young, $\mathrm{P}$.

1987. On Dividing an Amount According to Individual Claims or Liabilities. Mathematics of Operation Research, 12: 398-414.

Young, P.

1988. Distributive Justice in Taxation. Journal of Economic Theory, 43: 321-335.

Notas

1 Es posible que el retraso de numerosos proyectos hidráulicos se deba a este problema de control de las comunidades de agua.

2 Hay antecedentes de este comportamiento en el caso de la minería y los servicios sanitarios en relación con la agricultura en el Norte de Chile.

3 También se les denomina juegos de quiebra o juegos de bancarrota.
4 Hay aplicaciones de teoría de los juegos cooperativos para este tipo de problemas.

5 Como se sabe, en la religión judía juega un papel central la tradición oral que interpreta, codifica y comenta la Torá (que se supone de origen divino). Uno de los textos que transcribe la tradición oral es el Talmud que, por su sentido interpretativo del mensaje divino, no ha dejado de escribirse hasta nuestros días. Por esa razón los contenidos de este texto no se pueden fechar con precisión. 
